\title{
Co-counter Asymmetry in Fast Wave Heating and Current Drive
}

\author{
E.F. Jaeger, M.D. Carter, L.A. Berry, D. B. Batchelor, C.B. Forest,* \\ H. Weitzner**
}

Oak Ridge National Laboratory, Oak Ridge, Tennessee 37831-8071

*General Atomics, San Diego, California 92186-5608

**Courant Institute of Mathematical Sciences, New York University, New York, NY 10012

\begin{abstract}
Full wave ICRF coupling models show differences in plasma response when antenna arrays are phased to drive currents co and counter to the plasma current. The source of this difference lies in the natural up-down asymmetry of the antenna's radiated power spectrum. This asymmetry is due to Hall terms in the wave equation, and occurs even without a poloidal magnetic field. When a poloidal field is included, the up-down asymmetry acquires a toroidal component. The result is that plasma absorption (i.e. antenna loading) is shifted or skewed toward the co-current drive direction, independent of the direction of the magnetic field. When waves are launched to drive current counter to the plasma current, electron heating and current profiles are more peaked on axis, and this peaking becomes more pronounced at lower toroidal magnetic fields.
\end{abstract}

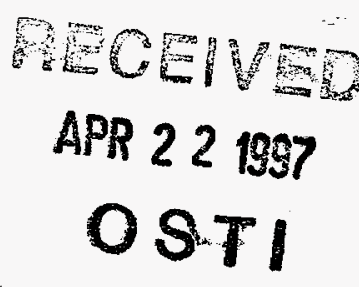

\section{INTRODUCTION}

Different plasma responses to neutral beam injection in directions co and counter to the plasma current have long been accepted as well understood in neutral beam heating of tokamaks (1). Similar differences have now been observed in electron heating and current drive experiments with toroidally phased antenna arrays in the ion cyclotron range of frequencies (ICRF) (2). At first, such differences are puzzling because nearly all radio frequency (RF) heating models are based on the homogeneous plasma dielectric tensor (3) which exhibits no obvious asymmetry for propagation in different longitudinal directions. But coupling of power between the antenna and the plasma is also a critical part of the problem, and it is here that the asymmetries become apparent. First, even for the homogeneous case, there are up-down (i.e. poloidal) asymmetries in wave propagation caused by the direction of Hall currents with respect to the applied magnetic field. This is observed as a shift in the poloidal spectrum of power radiated from the antenna in a magnetized plasma column with straight magnetic field lines (4). The shift is symmetric in the longitudinal wave number, but symmetry is broken when a poloidal magnetic field is applied. This was first noticed in the ST tokamak (5) when split eigenmodes were observed for each value of the toroidal wave number.

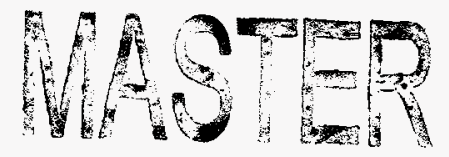




\section{DISCLAIMER}

This report was prepared as an account of work sponsored by an agency of the United States Government. Neither the United States Government nor any agency thereof, nor any of their employees, make any warranty, express or implied, or assumes any legal liability or responsibility for the accuracy, completeness, or usefulness of any information, apparatus, product, or process disclosed, or represents that its use would not infringe privately owned rights. Reference herein to any specific commercial product, process, or service by trade name, trademark, manufacturer, or otherwise does not necessarily constitute or imply its endorsement, recommendation, or favoring by the United States Government or any agency thereof. The views and opinions of authors expressed herein do not necessarily state or reflect those of the United States Government or any agency thereof. 


\section{DISCLAMMER}

Portions of this document may be illegible in electronic image products. Images are produced from the best available original document. 


\section{SYMMETRY OF THE COLD PLASMA WAVE EQUATION}

Consider a two dimensional (2-D) slab plasma in Cartesian coordinates with an external magnetic field $\mathbf{B}_{0}$ aligned along the $\mathrm{z}$ axis. Assuming that the component of the RF electric field parallel to $\mathbf{B}_{0}$ is zero [zero electron mass limit, (6)], and periodic dependence of the $R F$ fields in the $\mathrm{z}$ direction, a simple wave equation can be written for $\mathrm{B}_{\mathrm{z}}$, the RF magnetic field in the longitudinal direction,

$$
\left(\frac{y}{c}\right)^{2} B_{z}+i\left[\frac{\partial B_{z}}{\partial y} \frac{\partial}{\partial x}\left(\frac{K_{x}}{\left(K_{\perp}-n z\right)^{2}-K_{x}^{2}}\right)-\frac{\partial B_{z}}{\partial x} \partial \frac{K_{x}}{\partial y}\left(\frac{K_{\perp}^{2}}{\left(K_{\perp}-n\right)^{2}-K_{x}^{2}}\right)\right]+\nabla \cdot\left(\frac{\left(K_{\perp}-n z\right) \nabla B_{z}}{\left(K_{\perp}-n_{z}^{2}\right)^{2}-K_{x}^{2}}\right)=0
$$

The second term (in square brackets) is due to Hall currents that flow as a result of the $\mathbf{E} \times \mathbf{B}$ force, and is proportional to density and magnetic field gradients through the dielectric tensor element $K_{x}$. If $K_{x}$ and $K_{\perp}$ are real, Eq. (1) is invariant under the transformation $\mathrm{B}_{\mathrm{z}}(\mathrm{x}, \mathrm{y}) \Rightarrow \mathrm{B}_{\mathrm{z}}{ }^{*}(\mathrm{x},-\mathrm{y})$ or equivalently for the RF electric fields: $E_{\mathrm{x}}(\mathrm{x}, \mathrm{y}) \Rightarrow \mathrm{E}_{\mathrm{x}}{ }^{*}(\mathrm{x},-\mathrm{y})$ and $\mathrm{E}_{\mathrm{y}}(\mathrm{x}, \mathrm{y}) \Rightarrow-\mathrm{E}_{\mathrm{y}}{ }^{*}(\mathrm{x},-\mathrm{y})$, where $*$ denotes complex conjugate. The condition that $\mathrm{K}_{\mathrm{x}}$ and $\mathrm{K}_{\perp}$ are real is consistent with the usual cold plasma assumption of no collisions and therefore no absorption of wave energy. When collisions are included, $\mathrm{K}_{\mathrm{x}}$ and $\mathrm{K}_{\perp}$ become complex, and the up-down symmetry of Eq. (1) is destroyed.

These conclusions are born out by full-wave calculations with the PICES global wave code (7) including finite parallel RF electric field and warm plasma effects. The result in Fig. 1 is for a single toroidal harmonic, $n_{\zeta}=10$, with 32 poloidal $(\vartheta)$ harmonics and 100 radial $(\rho)$ mesh points. The geometry is that of a tokamak with no poloidal magnetic field and $\mathrm{B}_{0}=-2.0 \mathrm{~T}$ (into page), $\mathrm{R}_{\mathrm{axis}}=1.8 \mathrm{~m}$, $\mathrm{K}=1.9$ (elongation), $\mathrm{R}_{\text {plas- }}$ $\mathrm{ma}=2.31 \mathrm{~m}, \mathrm{f}$ $=60 \mathrm{MHz}$,
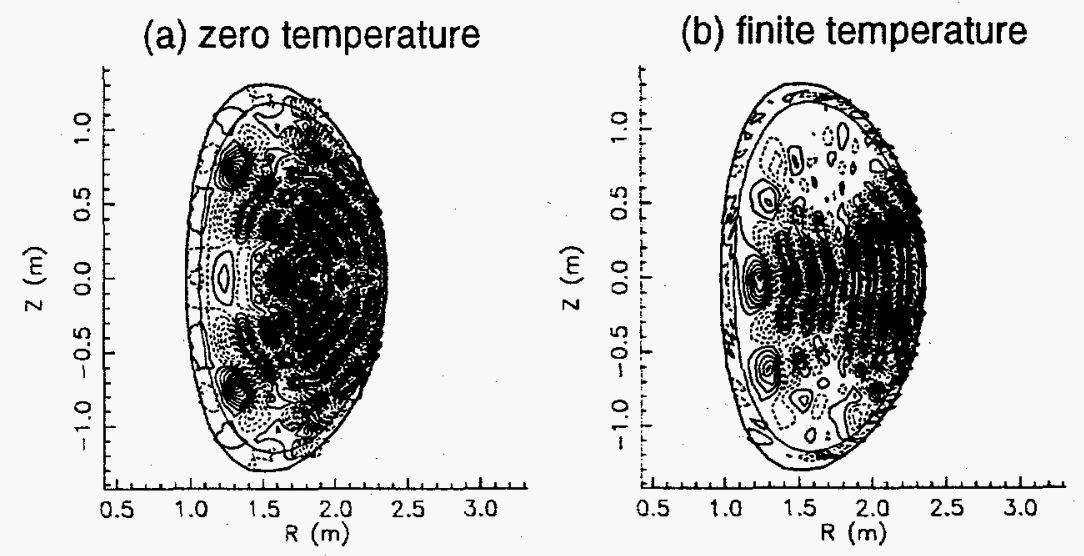

Figure 1. Wave electric field $\operatorname{Re}(\mathrm{E} \rho)$ from the PICES [7] for no poloidal magnetic field with (a) zero temperature and (b) finite temperature $(T e=20 \mathrm{keV}, T i=8 \mathrm{keV}$ )

and $\mathrm{n}_{\mathrm{e}, 0}=3.0 \times 10^{19} \mathrm{~m}^{-3}$. The majority ion species is deuterium with a $2 \%$ minority fraction of hydrogen. Results are calculated for (a) zero temperature and (b) finite temperature. $E_{\rho}$ and $E_{\vartheta}$ correspond to $E_{x}$ and $E_{y}$, respectively, in the slab model. Note the perfect up-down symmetry of $E_{\rho}$ in (a) without absorption. When ab- 
sorption is included in (b) through finite temperature, the up-down symmetry is destroyed. A plot of the radiated power spectrum in front of the antenna shows that the direction of energy flow in (b) is down, and reversing the sign of $\mathbf{B}_{\mathbf{0}}$ reverses the direction of energy flow.

\section{CO-COUNTER ASYMMETRY}

When a poloidal magnetic field is introduced, the up-down asymmetry described above acquires a toroidal component. In this case, the plane of the magnetic field in front of the antenna is tilted due to the pitch of the field lines. For a positive plasma current, the downward direction of energy flow in Fig 1(b) now has a toroidal component in the $-\zeta$ or "co" current drive direction. [Note that because of the negative electron charge, waves traveling in the $-\zeta$ direction, drive current in the $+\zeta$ direction]. Thus, an antenna which is phased to launch waves in the $-\zeta$ direction transfers more energy to the plasma (loads better) than the same antenna phased to launch in the $+\varsigma$ direction. This is in fact the case as shown by Fig. 2(a) where the spectrum of absorbed power is plotted versus toroidal mode number for $\mathrm{B}_{\varsigma}<0$ and $\mathrm{B}_{\vartheta}>0$. The calculation includes 100 uniformly excited toroidal modes. Parameters are those expected for the National Spherical Tokamak Experiment (NSTX) (8): $B_{0}=-0.30 \mathrm{~T}, \mathrm{R}_{0}=0.8 \mathrm{~m}, \mathrm{I}_{\mathrm{p}} \leq 1 \mathrm{MA}, \mathrm{K}=2.0$ (elongation), $\mathrm{R}_{\text {wall }}=1.39 \mathrm{~m}, \mathrm{f}$ $=41 \mathrm{MHz}, \mathrm{n}_{\mathrm{e}, 0}=3.0 \times 10^{19} \mathrm{~m}^{-3}, \mathrm{~T}_{\mathrm{e}, 0}=2.0 \mathrm{keV}, \mathrm{T}_{\mathrm{i}, 0}=1.0 \mathrm{keV}$. In NSTX, the co-counter asymmetry is particularly strong because of the large poloidal magnetic field near the antenna. When the sign of $\mathrm{B}_{\vartheta}$ is reversed in Fig 2(b), the asymmetry
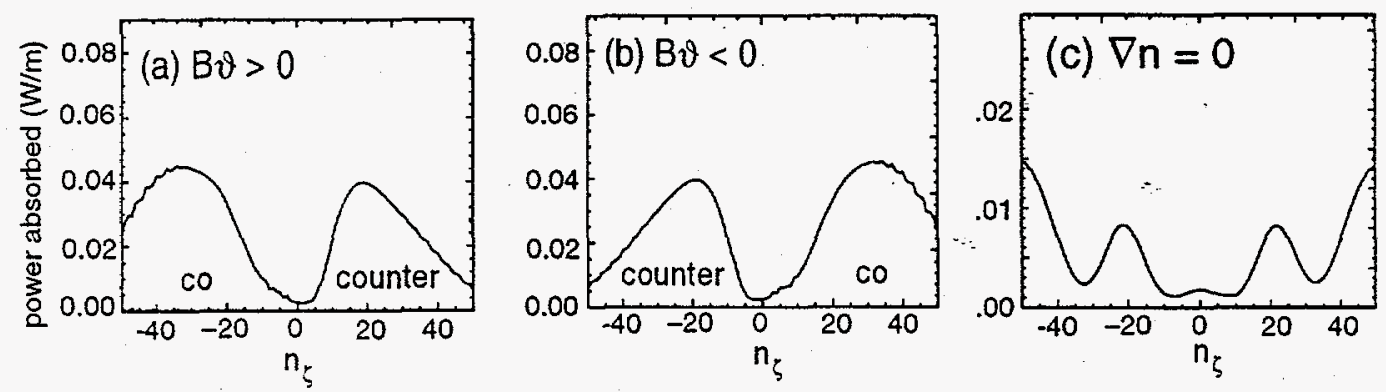

Figure 2. Spectrum of absorbed power in NSTX with $\mathrm{B} \zeta<0$ and (a) $\mathrm{B} \vartheta>0$. In (b), $B \vartheta<0$ and in (c), the density gradient is set to zero.

is also reversed. But in this case, the plasma current also changes sign so that co and counter directions reverse, and loading remains highest for the "co" direction. The general conclusion is that plasma absorption and hence antenna loading are always shifted or skewed toward the co-current drive direction independent of the direction of the applied magnetic field. When density gradients are forced to zero in Fig. 2(c), both up-down and co-counter asymmetries are eliminated because the 
Hall terms in Eq. (1) are proportional to density gradients. These results are obvious in the full-wave calculations of Fig.2, but they would not be seen in ray tracing calculations unless the rays were launched with a spectrum which models the updown asymmetry.

Current drive efficiency is also affected by the direction that waves are launched relative to the plasma current. Figure 3 shows radial profiles of current density $\mathrm{J}(\rho)$
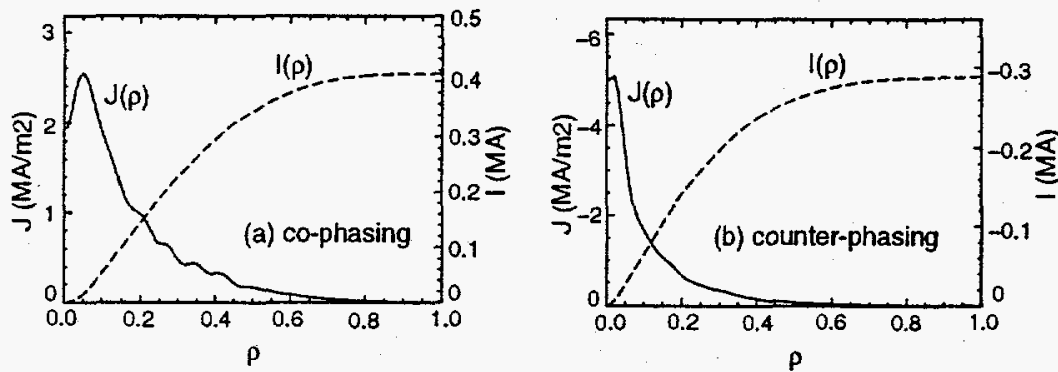
and integrated current $I(\rho)$ for the example of Fig. 1 but including a positive poloidal magnetic field. Although the total current Figure 3. Driven current profiles for the case of Fig. 1 but with a positive is slightly higher poloidal magnetic field and a smaller toroidal magnetic field $(B=-1.7 \mathrm{~T})$ for co-phasing in (a) co-phasing; (b) counter-phasing.

(a), the current density profile is more peaked near the axis for counter-phasing in (b). This peaking is more pronounced at low toroidal magnetic fields because the Hall terms in Eq. (1) are proportional to the magnitude of the $\mathbf{E} \times \mathbf{B}$ drift velocity or $E$ / $B$.

\section{ACKNOWLEDGMENTS}

The authors wish to thank M. Murakami, Y.R. Lin-Liu, R. Prater and P. E. Moroz for helpful discussions. This work is sponsored by Oak Ridge National Laboratory, managed by Lockheed Martin Energy Research Corp. for the U.S. Department of Energy under contract number DE-AC05-960R22464.

\section{REFERENCES}

1. Rome, J.A., Callen, J.D., Clarke, J.F., Nucl. Fusion 14 (1974) 141.

2. Prater, R., et. al., "Fast Wave Heating and Current Drive in DIII-D in Discharges with Negative Central Shear" in 16th. IAEA Fusion Energy Conference, Montreal, Canada, 7-11, October 1996.

3. Stix, T. H., "Waves in Plasmas", American Institute of Physics, New York (1992)

4. Messiaen, A. M., et. al., Nucl. Fusion 15 (1975) 75.

5. Adam, J., et. al., "Wave Generation and Heating in the ST-Tokamak at the Fundamental and Harmonic Ion Cyclotron Frequencies", in Sth Conf. on Plasma Physics and Controlled Nuclear Fusion Research, Tokyo, IAEA-CN-33/A3-2, 1974.

6. Jaeger, E. F., Batchelor, D. B., Weitzner, H., Whealton, J. H., Comput. Phys. Commun. 40 (1986) 33. (1993) 179.

7. Jaeger, E. F., Batchelor, D. B., Stallings, D. C., Nucl. fusion 33 (1993) 179.

8. Ono, M., Phys. Plasmas 2 (1995) 4075. 